\title{
Ist Chloroform ein geeignetes Gegenmittel nach Einatmung nitroser Gase?')
}

Von Dr. F. Curschmann, Greppin-Werke.

Unter der Bezeichnung ,nitrose Gase“ versteht man ein Gemisch von salpetriger Säure, Untersalpetersäure und Stickstoffoxyden. Es ist nun bekannt, daß ihre Einatmung, selbst in größerer Menge, für' den Menschen völlig unschädlich sein kann. Besonders von Chemikeın, die in Laboratorien arbeiteten, aber auch bei Betriebsunfällen damit in Berührung kamen, wird wiederholt angegeben, daß sie sehr wohl den seifigen Gesclimack, der nach der Einatniung dieser Gase auf der Zunge entsteht, verspürt hätten, aber sonst völlig unbehelligt geblieben wären. Und jeder, der Gelegenheit hat, bei Betriebsunfällen Leute zu sehen, die zweifellos größere Mengen nitroser Gase eingeatniet hatten, hat beobachtet, da $\beta$ dies gelegentlich ohne jede nachfolgende Gesundheitsschädigung geschah. Demgegeniiber stehen aber nun doch eine größere Anzahl von Fällen, bei denen ihre Einatmung, selbst wenn sie auch nur kurze Zeit und scheinbar nicht sehr intensiv erfolgte, schwere Erkrankungen, ja den Tod verursachte. Daraus geht hervor, daß die nitrosen Gase für den Körper nicht indifferent sind. Woran es aber liegt, daß sie es in dem einen Falle sind, im anderen nicht, vermag man aus den beschriebenen Fällen nicht zu sagen. Die Art ilırer Entstehung kann danach nicht das Ausschlaggebende sein, denn Gase, die auf gleiche Art entstanden, haben das eine Mal Erkrankungen hervorgerufen, das andere Mal nicht. Man kann auch nicht recht annehmen, daß ihre verschiedene Zusammensetzung die Wirkung beeinflusse. Daß die Erkrankten durch Schwächezustände besonders disponiert gewesen seien, geht aus den Berichten und Beobachtungen auch nicht hervor. Jedenfalls scheint die Disposition zu solchen Erkrankungen individuell sehr versehieden zu sein. Ich will nicht weiter auf die ältere, recht umfangreiche Literatur eingehen; jedenfalls ersieht man aber daraus, welches Interesse schon lange dieser Frage zugewendet wurde. Ganz besonders unheimlich erscheinen null die Dämpfe nicht nur deshalb, weil sie das eine Mal schädigen, das andele Mal nichit, sondern mit auch darum, weil die Wirkung oft erst llach Stunden eintritt, ohne da $\beta$ der Erkrankte vorher irgendwelche ihn beunruhigende Erscheinungen gehabt hat. Und auch in diesen Fällen kann der Ausgang tödlich sein.

Da nun in den verschiedensten Fabrikationszweigen die Möglichkeit der Entstehung dieser Gase bei Betriebsstörungen gegeben ist, hielt man es für richtig, daß jeder, der diese einzuatmen Gelegenheit liatte, Gegenmittel anwandte. So empfahl denn auch die chemische Berufsgenossenschaft im Jahre 1903 jedem, del nitrose Gave eingeatmet hatte, mehrmals 3 bis 5 Tropfen Chloroform, in ein Glas Wasser gegossen, zu geben. Zurückzuführen war diese Anordnung auf die Angabe eines Dr. Woisskopf in Johamnesburg, dessen Mitteilungen dann von Dr. Seyfferth, dem Direktor der Rheinisch-westfälischen Sprengstoff-A.-G., aufgegriffen und, wie er berichtet, mit gutenı Erfolg verwertet worden sind. Beide glauben, durch das Chloroform die Krämpfe, die nach ihrer Ansicht durch die Einatmung nitroser Gase entständen, zu beseitigen.

1) Vortrag, gehalten auf der Konferenz der Fabrikärzte der Chemischen Industrie. 
Eine wissenschaftliche Prïfung der Angelegenheit hat meines Wissens noch nicht stattgefunden. Wolıl sind aber schon von verschiedenen Seiten Bedenken gegen diese Vethode gcäußert worden, einmal, daß es doch nicht einwandfrei sei, cin Medikanent wie Chloroforn denl Gebrauch von Laien zu ïberlassen (Brat 1), dann aber auch, daß seine Wirkung in diesem Falle doch durch nichts bewiesen sei. Trotzden wurde die Vorschrift aber seither beibehalten, venututlich, weil man nichts Besseres an ihre Stelle zu sctzen wußte.

Es erschien mir daher nicht undankbar, die Sache experiunentell und an der Hand der vorhandenen Literatur genauer zu prüfen. Dabei glabube ich, nuir zwei Fragen vorlegen zll müssen, erstens, wie dic Wirkung der nitresen Gase in Körper sci, und zweitens, wic Chloroform, innertich in don vorgeschricbenen Gaben genonmen, wirken müsse. Daraus mußte sich von selbst ergeben, ob nian sich davon irgendwelchen Erfolg verspiechen konute oder nicht.

Inder Beschreibung des allge meinen Krankhoitsbildes sind sich alle Beobachter und Autoren einig. In leichten Fitlen tritt bei der Einatmung ein heftiger Hustemeiz auf, dem eine leichte Bronchitis folgen kann. In den schweren Fällen pflegen nach ciner ganz beschwerdefreien Zeit von einigen Stunden (bis 24) Atennot, Zyanose, blutiger Ausw urf, Unregelmäßigkeiten in der Herztätigkeit sich zu zeigen. Objektiv werden Volunlen auctum der Lunge und zahlreiche Rasselgeräusche beobachtet. Dicser Zustand kann $30-40$ stunden dauem, dann alhı̈̈hlich sich bessem oder aber unter den Zeichen des Luftmangels ad exitunn führen. Als bezeichnend wird von allen Beobachtern angeführt, daB das Bewußtsein meist bis zum Ende erhalten bleibe. Von Kränpfen, wie sic in Tierversuch (Eulenberg) (2) sich zeigen sollen, wird nic etwas berichtet; es scheint also das Zcntralnervensystem nicht wesentlich in Mitleidenschaft gezogen zu werden. Selbst wenn aher diese gefälntichen Zustände nicht letal cndigen, pflegen öfters schwere Gesundheitsschädigungen zur ïckzubleiben. Die oft schon während des Anfalles beobachtete Dilatation des Herzens, verbunden nit dessen unregelnä̈Biger Tätigkeit. bleibt meist auf dic Daucr bestehen, und es scheint, als ob der Helzulushel eine Entartung durch das Gift crleide (Schwerin) (3). Daneben pflegen die bronchitischen Erscheinungen meist auch nicht zu verschw inden, in deren Folge häufig Asthmianfälle, Neigungen zu Pneumonien, selbst 'Tuberkulose und dergleichen brobachtet werden, sodak es den Verdacht erweckt, als ob in dicsen Fällen sich Narben an den durch die Gase geätzten Stellen des Lungengewcbes gcbildet hätten. Diese Beobachtungen werden nir speziell von Praktikern (Bachfeld (4), Floret (5), Hahn) (6) bestätigt. Auch länger anhaltende Nierenreizungen, ja Entzündungen der Niere können zurüickbleiben. die auch auf cinc degenerative Wirkung der Gase schließen lassen. Leider liegen menschliche Sektionsbefunde nur wenige vor, so von A. Fränkel (7), Bauer (9), Künne (10), Savels (11), Schmicden (12). Holtzmann (13) und besonders auch von Ko(kel. ${ }^{1}$ ) Bci Fränkel hat es sich um Erecheinungen gehandelt, die er als Bronchiolitis fibrinosa obliterans bezeichnet und die ihre Ursache in der Vor̈̈tzung der Auskleidung der Bronchiolen durch dic Gase liaben. Kockel fand ähnlich in scinen wenige Stunden nach der Einatıung tödlich verlaufenen Falle einen hochgradigen Katarrh der Luftwege, akute Hyperänie und Oeden der Lunge. Auch seine Tierversuche bestätigten diesen Befund, daß es nacl nur kuızdauernder Einatmung nitroser Gasc selbst zu Bronchopneunonie kommen kamn. Auffallend waren auch die hyalinen Thromben, die er ebenso wic Bauer in den Lungenkapillaren fand. Aehnliche Beobachtungen im Tierversuch hatten vor ihn chon Lassar (14) und spiegel (15) gemaclat. Deren Arbeiten sind dcslialb vor besondcrem Intcresse, weil sie sich mit der Lösung der Frage befassen, ob die Wirkung der Gase eine rein lokile auf dic Atmungsorgane sei, oder ob es sich un eine Allgeneinvergiftung des. Körpers durch sic handeln könnc, also kuız, ob die Gase respirabel cder irrespirabel seien Neben Eulenborg haben die verschiedensten Autorën außer den schon genannten (Brat, Kobert (16), Kunkel (17) u. a.) nach dieser Richtung hin

1) Die Arbeiten von Risel und Loeschke wurden nuir erst während des Druckes bekanrt. experimentell gearbeitct. Und auch ich habe zallhleiche Tierversuche zu dicsen Zwecke angestchlt.

Wenn, wie das die Ansicht auch von Lowin (18) und Kobert ist, nitrose Gase respirabel sind, so müßten sie oder ihre Wirkung notgedrungen im Blute nachweisbar sein. Leider sind die Angaben in den beobachteten Fälen menschlicher Erkıankungen nach dicser Richtung liin ganz ungenau. Meist findet man nur angegcben, daß das Blut bräunlich gewesen sei. Spektroskopische Untersuchungen sind nie veröffentlicht worden. Gingen die Gase nun ins Blut ïber, so könnten sie darin nur als salpetrigsaures Natron auftreten; ind dieses ist ein bekannter Vethännglobinbildner (Bins) (19). Es nüßBten also nitrose Gase, falls sie iespirabel wäıen, das Oxylı̈̈moglobin des Blutes in Methänoglobin umwandch und als solches nachwcisbar sein. Damit ginge in schweren Fïllen die Herabsetzung der Alkaleszenz des Blutes einher, beides Veränderungen, die schwere Allgenteinerscheinungen, und daneben auch eine nachträgliche Alteration des Aufbaues der Körperorgane hervorrufen mußten.

Methänoglobin ist nun in den kasuistischen Berichten in. der Literatul weder bein Menschen noch bei Tieren bis jetzt je baschrieben worden. Wenigstens habe ich in keiner der kasuistischen oder experimentellen Arbeiten darüber eine Angabe entdecken können. Nur eine mündliche Mitteilung konnte ich von Schwerin erhalten, der in einen schr ernsten Falle in Anfang der Vergiftung diese Verëndcrung des Blutfarbstoffes sah. In Verlaufe der Krankhcit verschwand sie mit der allgenıeinen Besserung. Trotzden starb nach cinigen Tagen der Kranke an Herzschwäche.') Als einzige mitgeteilte Veränderung des Bhutes wird sonst nur wiederholt die brëunliche bis schwarze Farbe angegeben, und auch sie soll sich nach Kockels Ansicht nur dann finden, wenn das Tier in der giftigen Atmosphäre verendet ist. Nebenher halten einige Beobachter dic Unlwandlung des Oxyhämoglobins in Hänatin für erwiesen, dessen Spektıun anch beobachtet w urde (Kockel), andere (Harnack) sprechen von Acidhämog'obin. In meinen Versuclıen kam cs mir nun lediglich darauf an, die Blutbeschaffenheit nach der Einatmung zu studieren, da die lokalen Erscheinungen durch die Arbeiten von Fränkel und Kockel ja hinreichend geklärt sind. Die Alkaleszenzhorabsetzung, die nach den Arbciten von Lassar (24) und Frcudberg (25) eine uncrhebliche ist, liabe ich auBer acht gelassen. Bei den Vcrsuchen wollte ich nun einesteils die Verhältnisse, unter denen in Betriebe Leute zur Einatmung nitroser Gase kommen können, nöglichst getıeu nachahmen, anderseits aber auch dadurch, daß ich densclben Versuch unter stets gleichen Bedingungen öfters wiederholte, untersuchen, ob der eimnal beobachtete Befund konstant sci. ${ }^{2}$ ) In der Tat war dies der Fall.

Danach kaun uan wolla das Folgende sagen: 1. Bringt man cin Tier in einen Raum, dessen Luft scho⿰ eine größere hienge nitroser Gase enthält, oder füilut man dic Gase so rasch zı1, daß eine schädliche Venu. reinigung der Einatmungsluft sehr rasch erreicht wircl, so sind die nitrosen Gase respirabcl und wandeln das Oxyläulugglobin des Blutes, dus stets intra vitam noch entnommen wurde, in Mietlämoglobin un. Das Niet. hämoglobinspektrum ist stets deutlich sichtbar gewesen, und auf Zusat\% von Schwefelammonium zu den Blutlösungen ist stets das Spekt um des reduzierten Viethämoglobins erschienen. Das Blut gelaun leichter wie ronst. Bei (ler nikroskopischen Blutuntersuchung fiel mir cuff, daß die Erythrozyten schwerer als sonst den Furbstoff aufnalimen. (Fürbungen nuch (Gieinsa und mit Ehrlichscher Triazidlösung.) Vielfach lager sie in (ieldrollen zusamıneı, ohne da $B$ ich annehmen kann, da B dies dureh Drack künstlich geschehen sei. Iłır Hämoglobingehalt schien stets herabgesetz1. Und immer war der Beginn von Formveränderungen an ihnen erkeınbar; gewöhnlich sah man deutliche Stechapfelformen, vereinzelt anch Eiı :chnürungen. Dic Leukozyten waren meist auffallend vermehrt. Fs i.t dies ein Bild, wie es Blut bietet, wenn der Farbstoff aus den Erytluo-

1) Auch in einem mir von der Berufsgenossenschaft der Chemischen Industrie mitgeteilten, tödlich verlaufencn Fall wurde bei der Sektion der Methämoglobinnachweis erbracht.

2) Die Versuchsanordnung war stets die folgende. Durch einen Glaskasteıl, in den das Tier gebracht wird, wird ein gleichnäßiger Luftstrom, (500 l pro Stunde) geblasen. Diesem wird ein Gemiseh nitroser Case beigennischt zu etwa $3-5 \%$. Die nitrosen Gase wurden stets so eutwickelt, daß $500 \mathrm{~g}$ einer 10 prozentigen Natriumnitritlösung iı 10 Minuten $100 \mathrm{~g}$ verdïnute Schwefelsäure zutropften. Es entstanden so in 4 . Minuten etwa $1 \mathrm{l}$ Gas. In der einen Versuchsreihe wurden nun die Tiere in den gut gelüfteten Kasten gebracht und nun erst mit der Gasentwicklung begonnen, in der anderen die Tiere aber erst eingesetzt, wenn der Kasten schon mit Gasen teilweise angefüllt war. 
zyten ausgelaugt und in Plasma zu Methämorlobin nmgewandelt wird. Aber daneben finden sich auch sehon die Anzeichen dcr beginnenden Degeneration

In allen Fällen erfolgte der Tod des Tieres bald nach der Herausnahıne aus der verunreinigten Atmosphäre, trotz Zuführung reichlicher Luft. Tiere, die beim Verlassen des Kastens noch relativ recht lebhaft waren, gingen in spätestens ein bis zwei Stunden untcr den Srmptomen höchster Atemnot zugrunde. Abgesehen von der Veränderung des Blutes bestand jedesmal Lungenödem, also auch eine lokale Aetzwirkung der Gase auf die Bronchiolen und Alveolen, wio sie eben Kockel schon beschrieben hat. Makroskopisch Thromben nachzuweisen, wie Bauer sic beschreibt, ist mir aber ebensowenig wic jenem gelungen. Anzeichen der Degeneration am Herzen oder den sonstigen Organen war nio er kennbar. Dazu war wohl auch der Krankheitsverlauf zu kurz.

2. Die lokale Wirkung war num in allen Versuchen der alleinige Befund, in denen das Tier in einen Raum mit frischer Luft gebracht wurde, in dem ganz langsam der Gehalt an nitrosen Gasen anstieg. Nie gelang es bei diesen Versuchen, selbst wenn die Titre subjektiv schon währenc des Versuches schwere Erscheinungen boten, Methämoglobin orler Hämatin zu erkennen. Das Blut war dann auch immer hellrot und nicht schwerer gerinnungsfähig. Auch nikroskopisch war es unverändert. Auffallend war nur der Blutbefund kurz vor dem Verenden. Zwischen den beiden Oxyhämoglobinstreifen war immer ein schwächercr Schatten sichthar, während das rote Spektrum ganz rein war. Jch glaube, dal darin sich die Ueberladung des Blutes mit $\mathrm{CO}_{2}$ äuBert. Der Verlauf der Erscheinungen war insofern interessant, als die T'iere gewöhn lich nach der Entnahme aus dem Kasten sich in frischer Luft zu erholen schienen, um dann nach mehreren Stunden, oft erst an andercn Tage wieder krank $\mathrm{zu}$ werden und dann unter ähnlichen Erscheinungen wie die anderen, aber langsamer ejnzugehen. Der Verlauf war also sehr ähn lich dem beim Vienschen beschriebenen. Und es ist mir nun olne weiteres erklärlich, warum in den menschlichen Sektionsberichten nie das Auf. treten von Vethäinoglobin angegeben ist. Es konnte ebensowenig wie in unseren letztgenannten Tierversuchen vorhanden sein. Die mensch. lichen Fälle, die der ersten Versuchsreihe entsprechen, sind eben sehr selten und werden woll so plötzlich letal verlaufen sein, daß Blutunter suchungen garnicht erst gemacht wurden. Der Tod wïrde anch nicl:t so rasch erfolgt sein, wenn die Gase nicht monentan die Lunge über flutet und jufolgedessen die Alveolarwände hätten durchdringen und so ins Blut hätten gelangen können. Fs ist also erfordorlich, daß die nitrosen Gase auf cinmal in starker Konzentration au einen großen 'Teil der Iunge einwirken, damit sie respirabc! sind. Wim wird nach meinen Versuchen sagen körnen, daß eine plötzliche Beimengung von etwa $5 \%$ Gasen zu dieseln Frgebnis genügt. Steigt a ber der Gasgehalt von Spuren beginnend erst zu dieser Höhe an so ist sicherlich im Anfang die Aetzwirkung vorherschend. Das Epithelieneiweiß an den getroffenen Stellen wird koaguliert, und die nekrotischen Selichten bilden ein Hemmnis für das Durcholringen der Gase zu den Kapillaren. Dadurch wird auch sofort eine heftige Dyspnoe und Lïftung der Lunge ausgelöst, die im Beginn, solange der Gasgehalt noch kein zu hoher ist, auch die Ueberladung der Lungenluft mit Kohlensänre abschwächt. Der Tod erfolgt dann aber, wenn die nekrotisiciten Epithelien sich in das Alveolarlumen hinein abstoßen und es zum Oedem der Lunge und zu bronchopneumonisehen Prozessen kommt. Bis zu deren Ausbildung können aber mehrere Stunden verge'len, in denen das 'Tier sich relativ wohl befindet. Denn der Gasaustausch kann durch die unversehrten Bronchiolen und Alveolen ja noch leidlich genügend sein In dem Alsgenblick, in dem dies aber durch deren Verlegung nicht inehr der Fall ist, wird die Atemnot auftreten, und man wird dann objektiv die Vergrößerung der Lunge nachweisen können. Die Folgen des Ausfallens eines Teiles der Lunge sind zunächst die Ueberladung des Blutes mit Kohlensäure, daneben aber auch die Erschwerung des kleinen Kreis laufes durch die erwciterten Lungenkapillaren, dic ihrerseits wicde die Verteilung des Blutes in der Iunge verlangsant. Also ein Circulus viliosus, dessen Resultat neben cler Schädigung und Ueberantingung des Herzens ein hochgradiger Saucrstoffmangel des Blutes soin muf. Verstärkt werden diese Beschwerden noch dadurch, daB die Whastizität der Lunge herabgesetzt ist. Diese Frscheinungen nehmen so zu, daf das Tier erstickt. Es ist also dann das Krankhcitsbild und selbst cier Tod clurch die lokale Schürigung hintcichend erklärt, oline daß man eine Allgemeinvergiftung, deren Fe'llen der negative Blutbofund ja ans beweist, zu Hilfe nehmen mïiste.

Ich glaube, man darf diese Versuchsergebnisse wohl in allgemeinen auf den Menschen übertragen, da die klinischen Erscheinungen in den Tierversuchen mit den beschriebench Fällen sehr übertinstimmen. Sind nur geringe Spuren der Gase eingeatmet worden, so werden eine leichte Hyperämie, vereinzelte Verätzungen mit den Erscheinungen eines Bronchialkatarrhes als Folge beobachtet werden. Gelangen aber größere Gasmengen zur Einatinung, so geschieht das gewölnlich bei der allmählichen Entstehung der Dämpfe. Dann worden die lokalen

Aetzwirkungen mit ihren Folgen das Krankheitsbild bcherrschen und den möglicherweise letalen Arsgang cder aber die Folgeerscheinungen bedingen. Wohl bei den meisten gewerblichen Fällen wird so die Schädigung zustande kommen. Von einer Vergiftung kann man dann allerdings nicht reden. Tritt aber ein Mensch in cinen. Raum, dessen Iuft mit den. Gasen geschwängert ist, eilend c.in., so kann er mit wonigen ticfen Atenzügen soviel Gase aufnehmen, daß $\epsilon$ s in der oben geschilderten Art zur Allgemeinvergiftung kommen. kinn. Diese Erkrankungen werden sich durch das Fehlen der Latenzzeit und den kürzcren Verlauf auszeichnen. Blutveränd(rung und dic Lungensehädigung werden in Vercine den raschen Ausgang bedingen.

Ein wirksames Gegenmittel nun nüßtc nach zwei Richtungen hin wirken. Fs müßte vernciden, daß durch die zur Lunge gelangten Gase weitere Schädigungen als die, die schon vorhanden sind, entstehen. Es nü̈Bte also zun z̈chst dic noch in der Alveolarluft enthaltenen Gase neutralisieren. Daneben abor wäre scine Aufgabe, die schon bestchenden. Schädigungen in ihrer Wirkung zu vermindcrn oder gar aufzuhcben. Wie wir sahen, sind es der zunehmende Mangel an Saucrstoff, dic Ueberladung mit $\mathrm{CO}_{2}$, unter Tmständen dic; Butveränderungen, die das Leben bedrohen. Um diesen Faktoren $z u$ begegnen, mübte man einmal den mangclnden Sauerstoff ersetzen, daneben aber fiir eine reichliche Entlïftung der Junge, dic durch die herabgesetzte Elastizität, die Verengung der Bronchiolen und Alveolen nicht ausgiebig ist, sorgen. Evtl. müßtc man den Körper im Kampi gegen die Noxe unterstützen. In den Fillc.n allgemeiner Vergiftung wär: zu versuchen, cine Regeneration des veränderten Blutfarbstoffes, wenn möglich, herbcizufïhren oder fïr die untergegangenen. Erythrozyten einen moglichst raschen Ersatz anzulegen.

Es ist mun die Frage, ob Chloroform diese Fordexung zu erfïllen vermag. Dazu ist es nötig, kurz seine Wirkung auf den menschlichen Körper, wenn es inncrlich genommen wird, zu betrachten. Es liegen darïber verschicdene Berichte vor: so sind von Hof mann (21) und spezicll 'Taylor (20) Studien iiber diesen Gegenstand veröflentlicht worden. Eine eingehende Zusammenfassung findet man bei Meyer (22) und Gottlieb (Experimentclle Pharnazie). Danach wikt Chloroform, inmerlich genommen, ähnlich wie seine inhalicrten Dämpfe, nur bedeutend langsaner. Auf Schleimhäuten ruft es zunächst ein unangenehmes Kältcgefühl mit nachfolgender Hyperänie, also einen Reis hervor. Dieser Fmpfindming folgt dann aber cine gewisse Gefiihllosigkeit. Erst in größeren Mengen genonmen, tritt neben Aetzwilkungen, besonders auf die Magenschleimhaut, die Allgemeinwilkung hervor. Bekanntlich reagieren die Zellen des Großhirns an empfindliclisten auf Chloroform, sodaß ein Erlöschen der Sensibilitat die erste Folge ist, wenn es in größeren Dosen in den Körper gelangt. Dann crst werden das Rückenmark und zuletzt erst das Zentrum für die Respiration in Mitleidenschaft gezogen. Eino Vominderung des Hustenreizes oder eine Beruhigung krampthafter Atnıng kann also erst in nahezu völliger Naıkose zustande kommen. Dazu bedarf es, innerlich genommen, einer gı̈ßßın Gabe, jedenfalls von mehreren Gramm. Damit nähert man sich aber rasoh der toxischen Dosis, die für dic innerliche Aufnahme, wenn auch noch nicht einwandfrei festgestellt, doch jedenfalls nicht mchr wie $10 \mathrm{~g}$ betragen wird (Taylor). Dementsprechend ist auch dic Maximaldosis auf $0,5 \mathrm{~g}$, die höchste Tagesdosis auf $1,5 \mathrm{~g} \mathrm{f} \cdot \mathrm{st}$ gesetzt. Die Ausscheidung des Chlorotorms aus dem Kölper erfolgt mun sehr rasch, zum großen Teil durch die Lungen. Gibt man den Inhalt eines c'er vorgeschriebenen Tropfgläschen, der $0,5 \mathrm{~g}$ Chloroform beträgt, auf etwa cine Stunde verteilt, und zwar alle zehn Minuten fün Tropfen, so wird die Einzelgabe; meincr Jeinung nach entweder garnicht wirken oder höhstcns jodesmal in Halse einen Hustenreiz hervorrufen. Eine ber uhigende Allgemeinwirkung auf die Respiration ist sie aber garnicht imstande auszuïben. Denu das zuerst aufgenommene Chloroform ist schon. wieder großenteils aus dem Körper ausgeschieden, bevor die letzten Gaben genommen werden, sodaß auch eine etwaige kumulierende Wirkung nicht zustande kommt. Aber selbst das Einnehnien des in einem Gläschen enthaltenen Chloroforms auf cinmal wïrde nicht einen Reiz oder Sehmo:z in den Atmungswegen beheben könnem. Ganz abgesehen davon, 
ist es ja aber auclı nocl dic Frage, ob eine solche Beruhigung erwünscht und heilsam ist. Beständen wirklich Kı:̈̈mpfe, die ałlerdings nie beobachtet sind, so wïrle nur cine richtige Narkose sie beseitigen können. Dabei ist aber dann noclı zu bedenken, daß diesc Krämpfe ja nur auftıet’’n können, wenn die nitrosen Gase in irgendeiner Form blutvcrändernd gewirkt haben oder im Blute zum Zentralnervensystem transportiert werden. Durch große Clloroformgaben, die ja auch in das Blut ïborgehen, wird dem Blute nun doch einc neuc Leistung und rventuclle Schädigung zugemutet. Auch mit Rïcksieltt auf die brobachteten Herzschädigungen scheint die Verabreichung von Chlonoform bedenklich. In einen Versuche, den wir so anstellten, daß wir ein T'icr erst etwa $10 \mathrm{~g}$ Chloroform mit Luft vermischt einatmen ließcn und es dann erst in etwas betäubtem Zustande, aber sonst noch munter den nitrosen Gasen aussetztelı, salien wir, daßs der Tod rascher eintrat als bei anderen, nicht nuit Chlorofor Beweis zu selıon, daß man nicht eine neue Sclı̈̈digung deı sehon brstehenden zufiigen soll. Woun man demgegenüiber einwenden wird, daß Ben\%ol z. B. boi Tieren, die vorher ein Narcoticum erhalten haben, nicht so giftig wirkt wir olıne dies (Raubousck) (23), so ist eben zu bedenken, daß Benzol kein Blutgift ist, sondern lediglich im Blute, olıne dies zu vorïndern, seinen Angriffspunkten, den Zellen des Nervensystems, zugefülurt wird.

Daß Clılorof́rı sehlicßlich auf die Metlä̈noglobinbildung ohne Einfluß ist, ist selbstrerständlich.

Jedenfalls geht aus alledenı hervor, daß Chloroform in der vorgeschriebenen Dosis nach der Finatmung nitroser Gase unter keinen Umständen weder in leichten noch in selwweren Fïllen von Nutzen scin kann, sondern im Gegenteil die Besclıwerden noclı zu steigern vermag. Aber selbst, wenn es so, wic man hoffte, reizlindernd wirkte, miiBte es als e in Felller angesehen werden, es anzuwend'n, weil es die als heilsam anzusehende Dyspnoe unterdrückte und so denı Körper den Kaııpf gegen die eingedrungene Schädigung el'sehwerte. Es ist dalıcr weiterlıin die Aufrechterhaltung der eingangs angefiihrten Vorseluift wissenschaftlich nicht gerechtfertigt, zumil durch ihure Anwendung andere wirksame Maßregeln verzögert werden können.

Sie kann um so eher aufgegeben werden, als wir licute in der Lage sind, Ersatz dafiir zu bicten. Wenn cr auel nieht in allen Fällen von Erfolg sein wird, entspriclit el immerhin den durch die Wirkung der Gase bedingten Indikationen auf das beste. Es sind dies die schon ron Brat und anderen einpfohlenen Sauerstoffinhalationen. Brat ist wohl der erste gewesen, der ihre Wilkung wissenschaftlich begi ündete und im bescnderen zeigte, daß gerade nach Einatmung nitroser Gase sie die einzelnen Schädigungen bekämpfen können. Der Sauerstoff wird unter Druck inhaliert, das Blut radurch maximal damit gesättigt, damit also einer Sauerstoffverarmung volgebeugt und gleichzeitig aber der Gehalt der Alvocolarluft an Sauerstoff gesteigert, soda $\beta$ auch bei geringem Luftgelıalt er in genügender Menge vorhanden ist. Aber selbst bei Blutveränderungen können die Inhalationen von Nutzen sein. Wenn sie auch nicht ohne weiteres dic Zurückbildung res Metlıäınoglobins in Oxyhämoglobin ermöglichen, so wcrlen sie doch die weitere Umwandlung von normalem Blutfarbstoff erschweren resp. verhindern können. Nicht vermögen sie aber, falls die Elastizität der Lunge weiter sinkt und die Atnung immer oberfüchlicher wird, die Atmung genügend zu vertiefen, uı dem Ueberhandnelımen der Kolılensäure entgegen zu arbeitcn. Hier miissen als wirksam die auftretende Dyspnoe und die heftigen Hustenstöße angeselıen werden, dic die Liiftung der Lungc erleirhtern. Empfehlenswert erscheint mir in dieser Bcziehung auch der schon von anderen empfohlene Aderlaß mit nachfolgender Kochsalzinfusion. Dadurch wird man den kleinen Kreislauf, der durch dic Hyperïnie der Lunge erselwwert ist, entlasten und gleichzeitig einen 'Teil der Kohlensiiure evtl. auch des Metlü̈inoglobins aus denı Blute entfernen. Das verdiunnte Blut wird begierig den ihı gebotenen Sauerstoff aufnelımen. Aber auch die Regeneration der zelligen Blutbestandteile wird dadurch besonder's angeregt werden, wie ja auch eine Rürkbildung des Methäınoglobins in dem verdiinnten uuıd stärker alkalischen Blute als möglich erwiesen ist. Glaubt man quïlcude Schmerzen und den Hustcnreiz linclemn zu müssen, so wird dies zweckmäßiger mit Morplium oder scinen Derivaten als nit Cliloroform geschelien. Doch wird clas ebenso wie der Aderlaß von dem Verlauf der Erkrankung abhängen und im Ermessen des Arztes liegen müssen. Keinesfalls darf ilne Anwentung den Laien iiberlassen bleiben. Ganz unbedenklich ist es aber, die Sauerstoffinlualationen durel ein nur cinigernaben mit dem Apparat vertrautes Personal aussfiilıren zu lassen. Iclı halte es daher für richtig, die Anweisung iiber das Verabreieken von Chleroform zuriickzuzichen und ohne Zeitverlust alle Lcute, die nitrose Gase eingeatmet laben, gleichgïltig, ob sie Beschwerden laben oder nicht, Sauerstoff wiederholt im Laufe der folgenden Stunden inhalieren zu lassen.

Schlußfolgerung. Nitrose Gase sind respirable Gase. Jedoch werden sie nur respiliert, wenn sie plötzlich in einer starken Konzentration ringeatmet werden. Somst ist ilıre Wirlkung hauptsürolich lokalätzend auf die Lunge. Chloroforı kann daher ein gecignetes Gegennittel für derartige Vergiftungen uicht sein, ja unter Umständen schädlich witken. Ein sehr geeignetes Gegennittel sind Sauerstoffithalationen, evtl. der Aderla 3.

Literatur: 1. Brat, Die Bedeutung der Sauerstoffinhatationen in der Gewerbehygiene in M. Michaelis, Sauerstofftherapie, - 2. Eulenberg, Die Lehre von dell schädlichen und giftigen Gasen. -3 . Schwerin, 4, Bachfeld, 5 . Floret, 6. $\mathrm{Hah}$. Persönliche Mitteilungen - 7. A Fränkel, Deutsche medizinische Wochenschrift 1904 - 8. Kockel, Vierteljahrsschrift für öf Tentliche Gesundheitspflege 1898. 9. Banter. Dïsseldorf 1905, Festschrift - 10. Kï n ne Deutsche medizinische Wochen. Bati er, Disseldor 195, Festschill. - 10. Kan ne, Deutsche medizinische Wochenschrift 1897 . -11 . Sa vels, Deutsche medizinische Wochenschrift 1910. - 12. Sch in ie den, Zentralblatt für innere Medizin 1892. - 13. Holtz mann, Konkordia 1908. 14. Lassar, Zeitschrift für physiologische Chemie, Bandl, 1877. - 15. Spiege 1, Dissertation Würzburg. - 16. Kobert, Lelurbuch der Intoxikationen. - 17. Kunkel Handbuch der Toxikologie. - 18. Lewin, Toxikologie. - 19. Binz, Archiv für experimentelle l'athologie und Pharmakologie 1881. - 20. Taylor, On poisons in relation to medical jurisprudence and medicine 1848 . 21 . gerichtlchen Medizin. - 22 . Meyer und Cottlieb, Experimentelle Pharmakologie. - 23. Rambousek, Verhandmingen des Kongresses für Cewerbekrankheiten 1910.4. Lassar, Archiv für physiologische Chemie 1874. - 25. A. Freud berg, Virchows Archiv, Band 125 . 